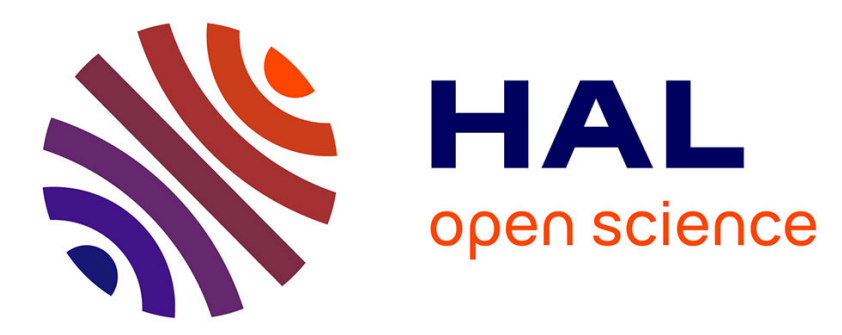

\title{
Experimental demonstration of the optical Helmholtz resonance
}

\author{
Paul Chevalier, Patrick Bouchon, Michael Verdun, Emilie Steveler, Nathalie \\ Bardou, Christophe Dupuis, Fabrice Pardo, Riad Haidar
}

\section{- To cite this version:}

Paul Chevalier, Patrick Bouchon, Michael Verdun, Emilie Steveler, Nathalie Bardou, et al.. Experimental demonstration of the optical Helmholtz resonance. Applied Physics Letters, 2018, 112 (17), pp.171110-1 - 171110-4. 10.1063/1.5028256 . hal-01853314

\section{HAL Id: hal-01853314 https://hal.science/hal-01853314}

Submitted on 3 Aug 2018

HAL is a multi-disciplinary open access archive for the deposit and dissemination of scientific research documents, whether they are published or not. The documents may come from teaching and research institutions in France or abroad, or from public or private research centers.
L'archive ouverte pluridisciplinaire HAL, est destinée au dépôt et à la diffusion de documents scientifiques de niveau recherche, publiés ou non, émanant des établissements d'enseignement et de recherche français ou étrangers, des laboratoires publics ou privés. 


\title{
Experimental demonstration of the optical Helmholtz resonance
}

\author{
Paul Chevalier, ${ }^{1,2}$ Patrick Bouchon, ${ }^{1,}$ a) Michael Verdun, ${ }^{1,2}$ Emilie Steveler, ${ }^{2}$ Nathalie Bardou, ${ }^{2}$ Christophe \\ Dupuis, ${ }^{2}$ Fabrice Pardo, ${ }^{2}$ and Riad Haïdar ${ }^{1,3}$ \\ 1) DOTA, ONERA, Université Paris-Saclay, F-91123 Palaiseau - France \\ ${ }^{2)}$ Centre de Nanosciences et de Nanotechnologies, CNRS, Université Paris-Sud, Université Paris-Saclay, \\ avenue de la Vauve, 91120 Palaiseau, France \\ 3) École Polytechnique, Département de Physique, 91128 Palaiseau, France
}

(Dated: 11 April 2018)

Optical nanoantennas are studied to manipulate light and enhance light matter interactions. Here, we experimentally demonstrate the optical Hemholtz resonance in a metallic slit-box structure, which is predicted to be harmonic and to enhance the electric field intensity by several orders of magnitude. It is fabricated thanks to a two steps electron beam lithography, between which the box was filled with benzocyclobutene (BCB). Up to $80 \%$ of the light is absorbed at a $\lambda=2.84 \mu \mathrm{m}$ wavelength under a beam focused by a Cassegrain objective $(\mathrm{NA}=0.4)$, even if the dimensions of this resonator are deeply subwavelength for both the slit (width $\lambda / 55$ and height $\lambda / 77$ ) and the box (width $\lambda / 7$ and height $\lambda / 37$ ). As expected from the inductance nature of the box, the optical properties of the BCB filling the box has no influence on the resonance behavior.

Optical nanoantennas are used to manipulate the light at a subwavelength scale ${ }^{1-4}$. They are able to generate strong field intensity enhancement in hot spots or hot volumes $^{5}$, which is an appealing feature for light matter interactions like non linear generation ${ }^{6}$, detection ${ }^{7}$ or thermal emission ${ }^{8}$. Several nanoantennas designs have been inspired by their radiofrequency alter egos, e.g. the Yagi-Uda antenna ${ }^{9}$, the bow-tie antenna ${ }^{10}$, the patch antenna $^{11}$. In these three examples, one of the dimension of the antenna is directly linked to its resonance wavelength, and these antennas exhibit harmonic resonances at lower wavelengths.

In contrast, the optical Helmholtz resonator was named by analogy with the $1 \mathrm{D}$ acoustic Helmholtz resonator $^{12}$. It consists of a metallic structure made of a box which is coupled to the semi infinite medium through a slit aperture ${ }^{13}$. Arrays of slits or boxes have been widely studied and are known to be the siege of Fabry Perot resonances $^{14}$, and likewise resonances are predicted to occurred in either the cavity or the aperture of the optical Helmholtz resonator. However, the Helmholtz resonance is predicted to appear at a higher wavelength than Fabry Perot resonances, which depends on each geometric parameter, and to be harmonic since it relies on an LC resonance mechanism ${ }^{13}$. It was suggested to be used for dual band absorption for infrared stealth applications ${ }^{15}$. Moreover, this structure has also been predicted theoretically to provide a strong field intensity enhancement ${ }^{16}$, which can be engineered from the near infrared to the $\mathrm{THz}$ wavelengths, and used for instance to enhance surface enhanced infrared absorption (SEIRA) ${ }^{17}$. Yet, there has been no experimental evidence of the Helmholtz resonance in optics, certainly due to the dimensions and geometry that are rather challenging to obtain.

In this letter, we report the experimental demonstration of the optical Hemholtz resonator in the mid-infrared

\footnotetext{
a)Electronic mail: patrick.bouchon@onera.fr
}
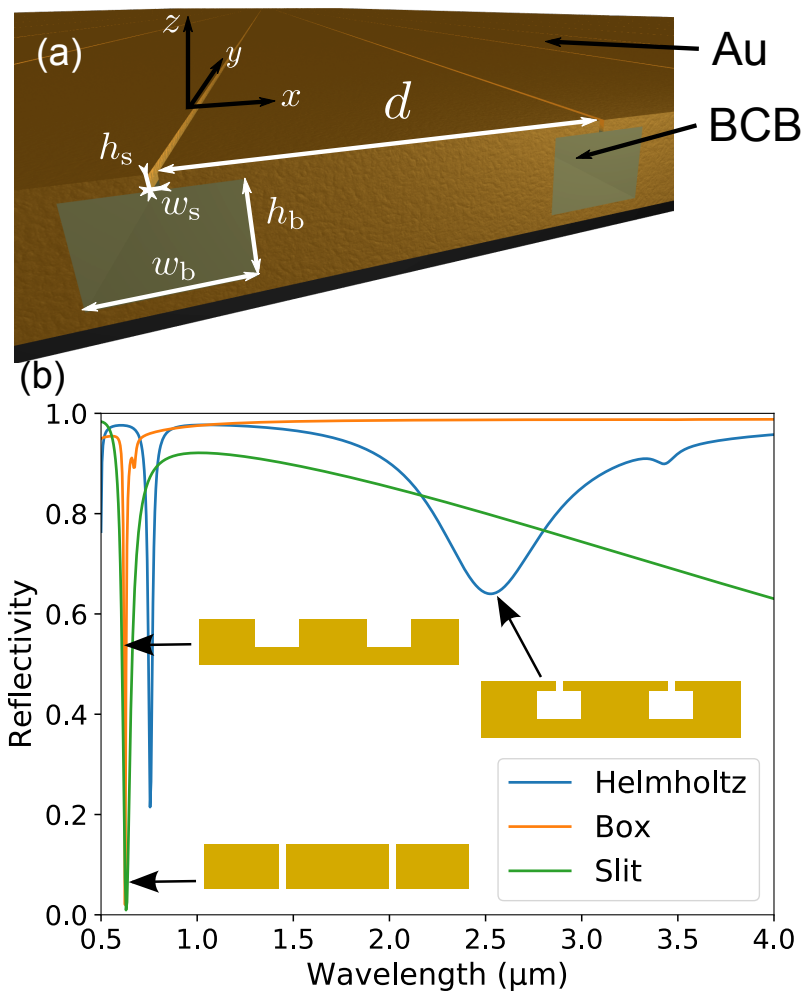

FIG. 1. (a) General schematic of the optical Helmholtz resonator, made of a box and a slit in metal, both infinite along the $y$ direction. (b) Computed reflectivity spectrum of the Helmholtz resonator array (period $d=450 \mathrm{~nm}$ ) made of a slit (width $w_{s}=52 \mathrm{~nm}$, height $h_{s}=37 \mathrm{~nm}$ ) and a box (width $w_{b}=400 \mathrm{~nm}$, height $h_{b}=77 \mathrm{~nm}$ ), compared with the reflectivity spectra of the array of slits (height $4 h_{s}$, width $w_{s}$, orange curve) and boxes (height $h_{b}$, width $w_{b}$,green curve). The impinging light is normally incident and TM-polarized.

range. We developed a process for fabricating the resonator using two levels of electron beam lithography, and a planarizing dielectric for filling the box as an intermediate step. The reflectivity measurement in TM polariza- 
(a)

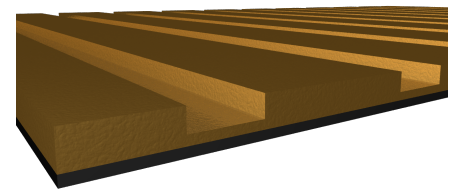

(b)

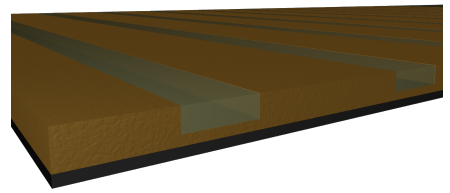

(c)

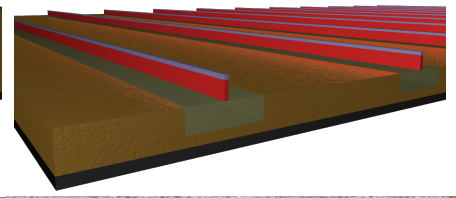

(d)

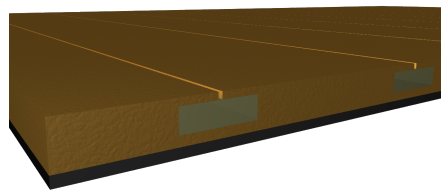

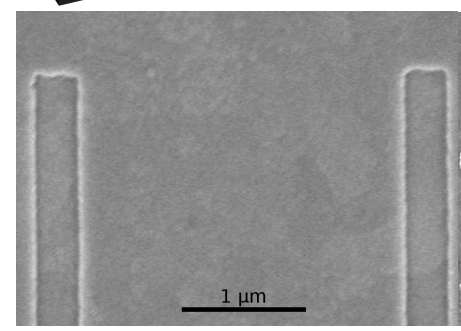
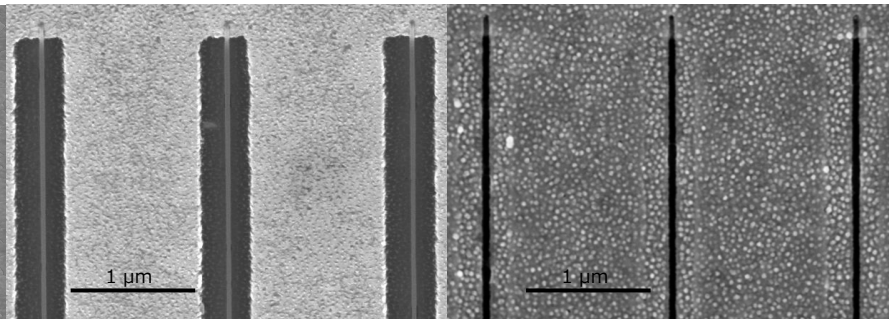

FIG. 2. Fabrication steps for a periodic grating of optical Helmholtz resonators with the corresponding top view SEM image: (a) Gold trenches are created by the lift-off of $80 \mathrm{~nm}$ of gold after a first e-beam lithography $(d=3 \mu \mathrm{m})$.(b) A dielectric $(\mathrm{BCB})$ is spin coated on the surface and etched by RIE to reach the gold surface $(d=3 \mu \mathrm{m})$. (c) A two layer lithography process is performed using PMMA and HSQ $(d=1.5 \mu \mathrm{m})$. (d) Gold is lifted-off resulting in the fabrication of the optical Helmholtz resonator $(d=1.5 \mu \mathrm{m})$. The narrow slits in the gold surface above the BCB-filled cavity, which is barely distinguishable.

tion under light focused by a Cassegrain objective showed a strong resonant absorption at the theoretically predicted wavelength, and a fair agreement is obtained between the experimental and the computed spectra. The slit is not perfectly centered in the middle of the box, and we show that this alignment offset induces an additional resonance at a lower wavelength.

The general shape of the optical Helmholtz resonators is recalled in Fig. 1(a). It is made of a box (height $h_{\mathrm{b}}$, width $w_{\mathrm{b}}$ ) closed by a narrow slit (height $h_{\mathrm{s}}$, width $w_{\mathrm{s}}$ ). In the following, the Helmholtz structure studied is long enough along the $y$-axis to be considered as infinite, and is repeated periodically along the $x$-axis with a period $d$. We choose to fill the cavity with benzocyclobutene (BCB), a planarizing dielectric, as shown in Fig. 1(a) to reduce the complexity of fabrication by avoiding freestanding parts, and to use gold as the metallic material for the rest of the structure. This structure was simulated using an exact Maxwell solver ${ }^{18,19}$, and the dielectric function of gold was computed using a Drude model which fits well the experimental data in the infrared ${ }^{20}$ : $\varepsilon_{\mathrm{Au}}(\lambda)=1-1 /\left[\lambda_{p} / \lambda\left(\lambda_{p} / \lambda+i \gamma\right)\right]$ with $\gamma=0.055$ and $\lambda_{p}=159 \mathrm{~nm}$. The BCB was described by a Lorentz model fitting experimental data ${ }^{21}$. The computed absorption spectrum, for a transverse magnetic (TM) polarized and normally incident light, is plotted in Fig. 1(b) for a given geometry $\left(w_{\mathrm{b}}=400 \mathrm{~nm}, h_{\mathrm{b}}=77 \mathrm{~nm}, w_{\mathrm{s}}=52 \mathrm{~nm}\right.$, $\left.h_{\mathrm{s}}=37 \mathrm{~nm}, d=450 \mathrm{~nm}\right)$. At $\lambda=2.53 \mu \mathrm{m}, 36 \%$ of the light is absorbed due to the Helmholtz resonance. Other resonances appear at lower wavelengths between $500 \mathrm{~nm}$ and $700 \mathrm{~nm}$. The small peak at $\lambda=3.4 \mu \mathrm{m}$ is due to a vibrational absorption of BCB that is enhanced by the Helmholtz resonator ${ }^{21}$. For the sake of comparison, the absorption spectra are also plotted under the same illumination conditions for an array of slits (height $4 h_{\mathrm{s}}$, width $w_{\mathrm{s}}$ ) and for an array of cavity filled with BCB (width $w_{\mathrm{b}}$, height $h_{\mathrm{b}}$ ) that also exhibit resonant absorption, but at lower wavelengths.

The key properties of the Helmholtz resonance, that distinguishes it from others, are: (i) A giant field enhancement, typically two orders of magnitude higher than expected with gap plasmons resonances. (ii) A single resonance behavior with no harmonics. (iii) No influence of the permittivity of the material filling the cavity on the resonance wavelength. (iv) The resonance wavelength $\lambda_{r}$ depends on the square root of all four geometrical parameters according to the following formula:

$$
\lambda_{r} \propto 2 \pi \sqrt{\varepsilon_{s} \frac{h_{b} w_{b} h_{s}}{w_{s}}},
$$

where $\varepsilon_{s}$ is the permittivity of the material filling the slit. Noteworthily, the permittivity of the material filling the cavity has no influence on the resonance wavelength. We now consider an Helmholtz structure with a larger period $d=1.5 \mu \mathrm{m}$, in order to be closer to the critical coupling of the resonator, and the following goal dimensions: $h_{\mathrm{b}}=80 \mathrm{~nm}, w_{\mathrm{b}}=400 \mathrm{~nm}, h_{\mathrm{s}}=40 \mathrm{~nm}, w_{\mathrm{s}}=50 \mathrm{~nm}$.

The fabrication process starts with the deposition of $20 \mathrm{~nm}$ of chrome followed by $200 \mathrm{~nm}$ of gold on a doped silicon wafer. A first step of e-beam lithography is performed to define the cavity using ma-N 2405 resist spun at $3000 \mathrm{rpm}$ on top of the gold layer. A grating pattern is written on a $0.5 \times 0.5 \mathrm{~mm}^{2}$ area thanks to a Vistec EBPG 5000 electron beam pattern generator at $100 \mathrm{kV}$. The resist is developed using a tetramethyl ammonium hydroxide (TMAH) based developer (Clariant AZ $826 \mathrm{MIF}$ ) for 30 seconds. Then $80 \mathrm{~nm}$ gold is evaporated onto the sample and lifted-off with acetone (see Fig. 2(a)) to fabricate trenches of height $80 \mathrm{~nm}$ and width $400 \mathrm{~nm}$. Benzocyclobutene (BCB) is spin coated on the sample and baked at $250^{\circ} \mathrm{C}$ for 3 hours under nitrogen 
flow. A reactive ion etching (RIE) plasma $\left(\mathrm{SF}_{6}+\mathrm{CHF}_{3}\right)$ is done so that the surface of the $\mathrm{BCB}$ reaches the gold surface while keeping the cavity filled with dielectric (see Fig. 2(a)). A scanning electron microscope (SEM) image of the structure at this step is shown in Fig. 2(b), showing the gold trenches (light grey) filled with a dielectric (black). On top of theses trenches, $150 \mathrm{~nm}$ of polymethyl methacrylate (PMMA) is spin coated and baked at $160^{\circ} \mathrm{C}$ degrees for 30 minutes and $60 \mathrm{~nm}$ of hydrogen silsesquioxane (HSQ) in spin-coated and baked on a hot plate at $110^{\circ} \mathrm{C}$ for 20 minutes. A $20 \mathrm{~nm}$ pattern is written at $100 \mathrm{kV}$. The resist is developed for 10 seconds in a potassium hydroxide $(\mathrm{KOH})$ based developer (Clariant AZ $400 \mathrm{~K}$ diluted at $50 \%$ ) and rinsed in distilled water. The PMMA is etched with a RIE $\mathrm{O}_{2}$ plasma for $60 \mathrm{sec}-$ onds to create a tall and thin wall of polymer centered on the cavity (see Fig. 2(c)). Finally $50 \mathrm{~nm}$ of gold is evaporated and lifted-off using trichloroethylen at $90^{\circ} \mathrm{C}$ in ultrasound bath, which terminates the process. A SEM image showing the slits from the top of the structure at this final step is shown in Fig. 2(d), on this image a slight variation of the contrast allow to distinguish the position of the underlying cavity. The choice of a bi-layer resist stack for the final lift-off of the slit is motivated by the need for a fine resolution of the pattern; also the RIE etching step using the HSQ as a mask allows to create an undercut of the PMMA resist ${ }^{22}$ which eases the following lift-off. The second level is aligned on the lower level with alignment markers positioned during the first step of lithography and detected by the e-beam pattern generator. The accuracy of this alignment on the sample size is about $50 \mathrm{~nm}$, and a slight shift of the slit in regard to the cavity can be expected.

The reflectivity of the fabricated sample was measured using a Bruker Vertex 70 Fourier Transform Infrared (FTIR) spectrometer, with the Bruker Hyperion 2000 microscope using a Newport Cassegrain reflective objective (15x and NA 0.4). The measurements were done using the internal mid-infrared source of the spectrometer and an InSb photodetector. A square area of $100 \mu \mathrm{m} \times 100 \mu \mathrm{m}$ is imaged with the microscope using knives and the beam is linearly-polarized with the magnetic field along the slits using an IR polarizer. In the following, we define the magnetic parallel polarization, for which the magnetic field is in the plane parallel to the orientation of the grating (plane yz, i.e., $H_{x}=0$ ), to avoid any confusion with the TM polarization. The light is in the magnetic parralel polarization after going through the polarizer and the Cassegrain objective, and is exciting the TM modes of the metallic structure. A schematic of the beam on the sample surface recalling the polarization is shown in Fig. 3(a).

The magnetic-parallel polarized measured reflectivity of this sample, plotted as a function of the wavelength in Fig. 3(b) (blue continuous line), exhibits two resonance wavelengths at $\lambda_{r}=1.56 \mu \mathrm{m}$ and $\lambda_{r}=2.82 \mu \mathrm{m}$. It should be underlined that light is incoming on a gold film patterned on less than $4 \%$ of its area, yet more than $70 \%$
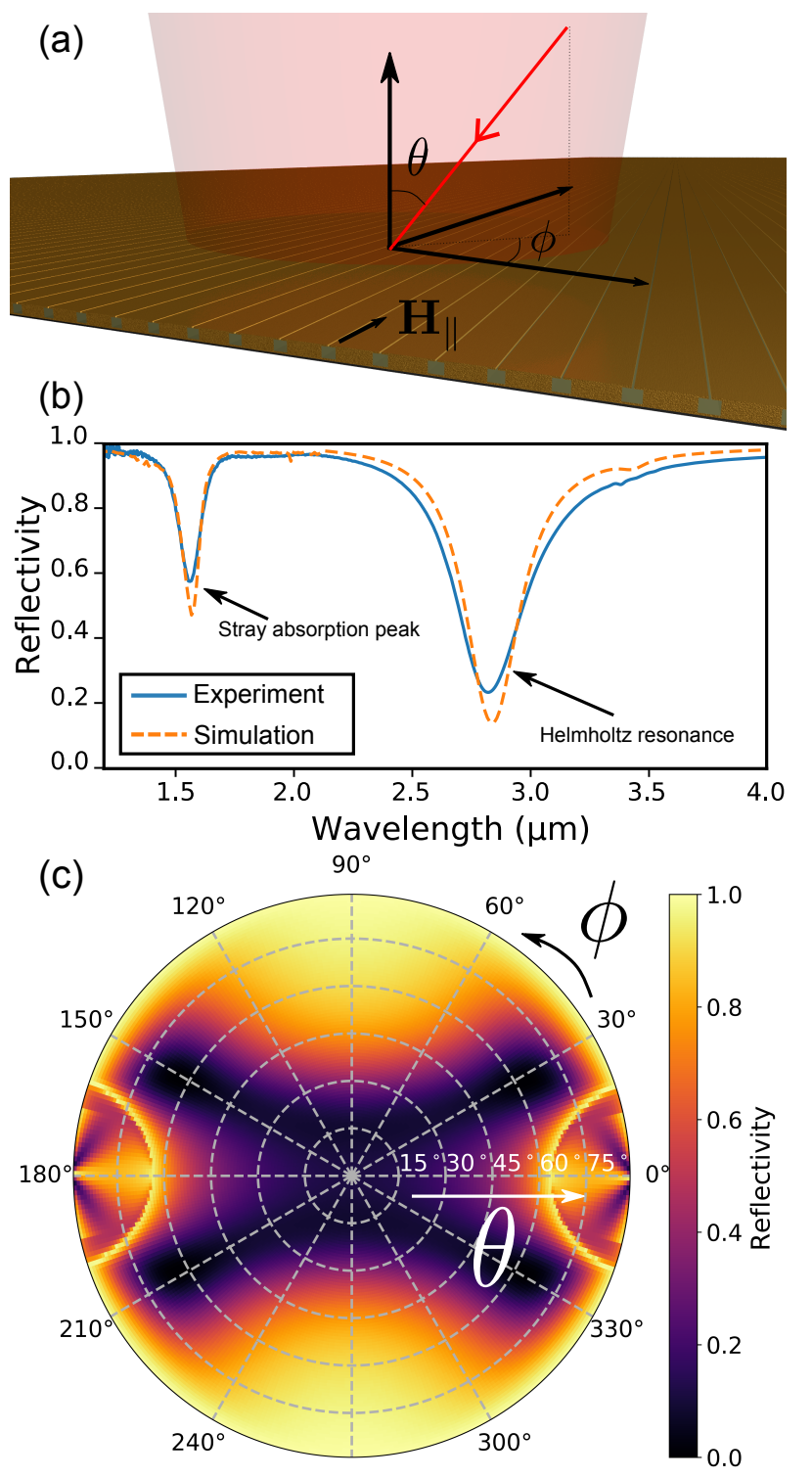

FIG. 3. (a) Schematic representation of the experimental measurement of reflectivity: the incident beam is polarized and focused by a $15 \mathrm{x}$ Cassegrain objective and the area of interest limited to a square area of $100 \mu \mathrm{m} \times 100 \mu \mathrm{m}$. (b) Reflectivity measurement of the structure with $h_{\mathrm{b}}=77 \mathrm{~nm}$, $w_{\mathrm{b}}=400 \mathrm{~nm}, h_{\mathrm{s}}=37 \mathrm{~nm}, w_{\mathrm{s}}=52 \mathrm{~nm}$ (blue line) under light magnetic-polarized focused by a Cassegrain objective with $\mathrm{NA}=0.4$. The simulated response is obtained by integrating the polar angle $\theta$ from $12^{\circ}$ to $23.6^{\circ}$ and the azimuthal angle $\phi$ from $0^{\circ}$ to $360^{\circ}$ (orange dashed line). (c) Computed angular diagram at the resonance wavelength $\lambda=2.84 \mathrm{\mu m}$ for a magnetic parallel polarized wave.

of the incoming light is absorbed at the higher resonance wavelength. The simulated response of a Helmholtz resonator is also plotted (orange dashed line) with the following dimensions: $h_{\mathrm{b}}=77 \mathrm{~nm}, w_{\mathrm{b}}=400 \mathrm{~nm}, h_{\mathrm{s}}=$ $37 \mathrm{~nm}, w_{\mathrm{s}}=52 \mathrm{~nm}$, and the slit was offset by $51 \mathrm{~nm}$ compared to the center of the box. The values taken for the box and slit widths, as well as the slit offset, 
are coherent with the SEM measurements (see Fig. 2). The simulated curve also corresponds to the integration of the reflectivity over various incidences: polar angles $\theta$ from $12^{\circ}$ to $23.6^{\circ}$ and azimuthal angles $\phi$ from $0^{\circ}$ to $360^{\circ}$ to take into account the microscope objective. A rather fair agreement is obtained between the computed and experimental spectra. As expected from Eq. 1, the resonance wavelength is close to the one predicted in Fig. 1 , although it is red-shifted. However, the level of reflectivity is different. This is due to both the change of the period that modifies the coupling factor with the continuum (i.e., the level of absorption at resonance), and the change of the incidence configuration. Eventually, there is the apparition of a second resonance that can be attributed to the slit offset. In the following, we theoretically investigate the influence of both the focalization and of the slit offset on the response of the Helhmoltz resonator.

To understand how the integration of the response over various incidences influence the resonance, we plotted on the polar diagram in Fig. 3(c) the reflectivity response at the resonance wavelength $(\lambda=2.82 \mu \mathrm{m})$ as a function of the conical angle of incidence. The Helmholtz resonance is localized, and for $\theta<25^{\circ}$, the absorption is higher than $75 \%$ for any incidence. It should be noted that for the polar angle values used by the Cassegrain objective, the azimuthal angle has a slight influence on the actual reflectivity, but this impacts mainly the quality factor of the resonance. Noteworthily, it has been shown that the angular tolerance of the Helmholtz resonator can be improved by inserting a high refractive index material inside the slit ${ }^{23}$.

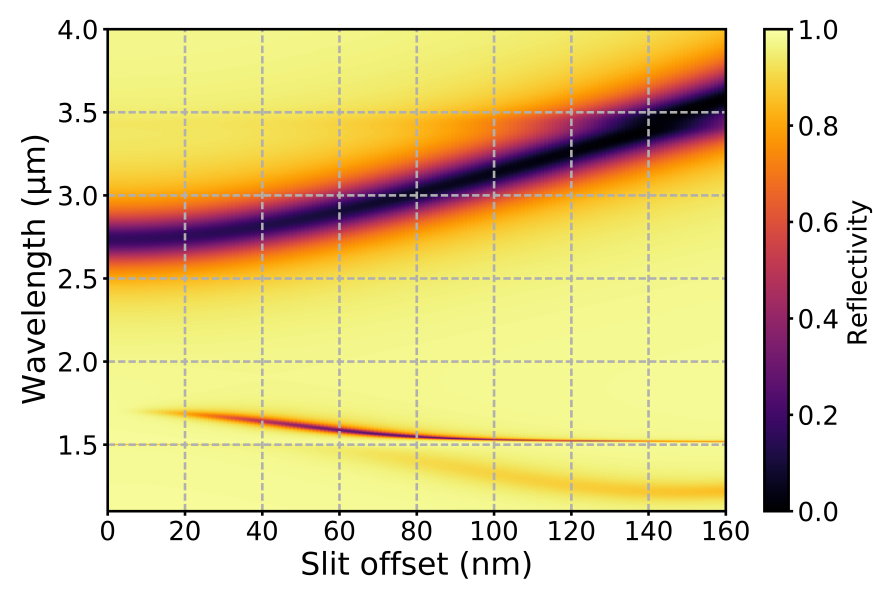

FIG. 4. Computed reflectivity diagram as a function of the wavelength and of the offset of alignment between the slit and the box. The reflectivity is computed for a TM-polarized normally incident plane wave.

In order to study the consequence of the misalignment between the slit and the box, we plotted in Fig. 4 the reflectivity spectrum of a Helmholtz structure with the slit offset. In the case of a perfectly centered slit, the reflectivity exhibits only a single resonance peak. As the slit moves away from the center of the structure, the main resonance is red-shifted, while a secondary resonance appear. The secondary resonance in the experimental curve of Fig. 3(b), and the red-shift of the Helmholtz resonance are indirect proofs that the slit was slightly misaligned during the fabrication process and it justifies the offset value used for the fit.

To conclude, a process to fabricate optical Helmholtz resonators has been presented, and used to experimentally demonstrate the optical Helmholtz resonance thanks to measurements in the near and mid-infrared were measured. A secondary resonance was measured at a lower wavelength, but it is explained by a defect on the symmetry of the resonator. The agreement between the theoretical and experimental far field optical properties of the resonators is a first step towards the experimental demonstration of the high field intensity enhancement ${ }^{16}$. An experimental proof could be given by measuring the enhancement of light matter interactions inside the slit, either by putting in it molecules with absorption features (SEIRA) or a nonlinear material.

\section{ACKNOWLEDGMENTS}

We acknowledge financial support from the ONERA through the MOLIERE project and from the Agence Nationale de la Recherche (ANR) (ANR-16-CE39-0007).

${ }^{1}$ J.-J. Greffet, Science 308, 1561 (2005).

${ }^{2}$ T. Teperik, F. De Abajo, A. Borisov, M. Abdelsalam, P. Bartlett, Y. Sugawara, and J. Baumberg, Nature photonics 2, 299 (2008). ${ }^{3}$ C. M. Watts, X. Liu, and W. J. Padilla, Advanced Materials 24, OP98 (2012)

${ }^{4}$ Y. Cui, Y. He, Y. Jin, F. Ding, L. Yang, Y. Ye, S. Zhong, Y. Lin, and S. He, arXiv preprint arXiv:1404.5695 (2014).

${ }^{5}$ J. A. Schuller, E. Barnard, W. Cai, Y. Jun, J. White, and M. Brongersma, Nature Materials 9, 193 (2010).

${ }^{6}$ H. Aouani, M. Rahmani, M. Navarro-Cía, and S. A. Maier, Nature nanotechnology 9, 290 (2014).

${ }^{7}$ M. W. Knight, H. Sobhani, P. Nordlander, and N. Halas, Science 332, 702 (2011).

${ }^{8}$ M. Makhsiyan, P. Bouchon, J. Jaeck, J.-L. Pelouard, and R. Haïdar, Applied Physics Letters 107, 251103 (2015).

${ }^{9}$ T. Kosako, Y. Kadoya, and H. F. Hofmann, Nature Photonics 4, $312(2010)$.

${ }^{10}$ S. Kim, J. Jin, Y.-J. Kim, I.-Y. Park, Y. Kim, and S.-W. Kim, Nature 453, 757 (2008).

${ }^{11}$ P. Bouchon, C. Koechlin, F. Pardo, R. Haïdar, and J. Pelouard, Optics Letters 37, 1038 (2012).

${ }^{12} \mathrm{H}$. von Helmholtz, Theorie der Luftschwingungen in Röhren mit offenen Enden, 80 (W. Engelmann, 1896).

${ }^{13}$ P. Chevalier, P. Bouchon, R. Haïdar, and F. Pardo, Applied Physics Letters 105, 071110 (2014).

${ }^{14}$ P. Bouchon, F. Pardo, B. Portier, L. Ferlazzo, P. Ghenuche, G. Dagher, C. Dupuis, N. Bardou, R. Haïdar, and J. Pelouard, Applied Physics Letters 98, 191109 (2011).

${ }^{15}$ C. Zhang, C. Huang, M. Pu, J. Song, Z. Zhao, X. Wu, and X. Luo, Scientific Reports 7, 5652 (2017).

${ }^{16}$ P. Chevalier, P. Bouchon, J. Greffet, J. Pelouard, R. Haïdar, and F. Pardo, Physical Review B 90, 195412 (2014).

${ }^{17}$ T. G. Mayerhöfer and J. Popp, Nanophotonics 7, 39 (2018).

${ }^{18}$ P. Bouchon, F. Pardo, R. Haidar, and J. Pelouard, JOSA A 27, 696 (2010). 
${ }^{19}$ P. Chevalier, P. Bouchon, F. Pardo, and R. Haïdar, Journal of the Optical Society of America A 31, 1692 (2014).

${ }^{20}$ R. L. Olmon, B. Slovick, T. W. Johnson, D. Shelton, S.-H. Oh, G. D. Boreman, and M. B. Raschke, Physical Review B 86, 235147 (2012).

${ }^{21}$ W. Folks, J. Ginn, D. Shelton, J. Tharp, and G. Boreman, physica status solidi (c) 5, 1113 (2008).

${ }^{22}$ A. E. Grigorescu and C. Hagen, Nanotechnology 20, 292001 (2009).

${ }^{23}$ P. Chevalier, P. Bouchon, E. Sakat, J.-L. Pelouard, F. Pardo, and R. Haïdar, Optics Letters 40, 2735 (2015). 